\title{
Effects of Recombinant Human Erythropoietin on Inflammatory Factors in Rats with Traumatic Brain Injury
}

\author{
KAI XUE* ${ }^{*}$ ZHENG JIN, BINBIN ZHANG, GUOQING HAN AND CHEN ZHANG \\ Department of Neurosurgery, Tianjin Huanhu Hospital, Tianjin 300350, China
}

\section{Xue et al.: Recombinant Human Erythropoietin and Traumatic Brain Injury}

\begin{abstract}
To study the effects of recombinant human erythropoietin on inflammatory factors in rats with traumatic brain injury is the main objective. A total of 45 specific-pathogen-free grade male Sprague-Dawley rats were randomly assigned into sham operation group (sham group), model group and recombinant human erythropoietin intervention group (treatment group) $(n=15)$. Model and treatment groups were prepared into traumatic brain injury model by hitting the head through the modified Feeney's free-fall impact method, while the head of sham group was not hit. After modeling, treatment group was intraperitoneally injected with recombinant human erythropoietin at $5000 \mathrm{IU} / \mathrm{kg}$ daily and sham and model groups were intraperitoneally injected with the same dose of normal saline. The rats were killed after $7 \mathbf{d}$ of continuous administration. The changes of brain mitochondrial membrane potential were detected through rhodamine 123 staining and immunocytochemistry and Western blotting were separately employed to measure the expressions of interleukin-1 $\beta$, interleukin- 6 and tumor necrosis factor- $\alpha$ in brain tissues and the expression levels of dynamin-related protein 1 , fission 1 , mitofusin 2 and optic atrophy 1 , mitochondrial dynamics related proteins in brain tissues. Compared with sham group, model group exhibited significantly weakened rhodamine 123 fluorescence intensity, increased expressions of interleukin-1 $\beta$, interleukin-6 and tumor necrosis factor- $\alpha$, dynamin-related protein 1 and fission 1 and reduced expressions of mitofusin 2 and optic atrophy 1 in brain tissues $(\mathbf{p}<\mathbf{0 . 0 5})$. In comparison with model group, treatment group had significantly enhanced rhodamine 123 fluorescence intensity, reduced expressions of interleukin-1ß, interleukin-6 and tumor necrosis factor- $\alpha$, dynamin-related protein 1 and fission 1 and elevated expressions of mitofusin 2 and optic atrophy 1 in brain tissues $(p<0.05)$. Recombinant human erythropoietin can protect the brain after traumatic brain injury by relieving the inflammatory response and mitochondrial injury after traumatic brain injury.
\end{abstract}

Key words: Recombinant human erythropoietin, traumatic brain injury, inflammatory response, mitochondrial injury

Traumatic brain injury (TBI), one of the most common neurosurgical diseases in clinic $^{[1]}$, has such major clinical manifestations as dizziness, headache or disturbance of consciousness, which further induces various neurological dysfunction, movement disorders and even loss of self-care ability ${ }^{[2]}$. Currently, TBI is mainly caused by traffic accidents and violent incidents. It exhibits high fatality and disability rates in the later period, thus bringing a heavy burden to the patient's family and the society. Thus, discovering reliable treatment approaches to restore the neurological function and improve the survival rate of patients has become a research priority in the field of neurosurgery. The inflammatory response of brain tissues after TBI is the most important pathological change in the later

*Address for correspondence E-mail: 766364170@qq.com

May-June 2021 progression of disease ${ }^{[3]}$. Hinson et al. ${ }^{[4]}$ revealed that the mitochondrial dysfunction of nerve cells after TBI participated in the whole pathologic evolution after brain injury. Hence, looking for drugs to control the inflammatory response and reduce or relieve the mitochondrial dysfunction of nerve cells from the current neurosurgery treatment methods has become an effective way to control the progression of $\mathrm{TBI}^{[5]}$. Recombinant human erythropoietin (rhEPO) is a drug

This is an open access article distributed under the terms of the Creative Commons Attribution-NonCommercial-ShareAlike 3.0 License, which allows others to remix, tweak, and build upon the work non-commercially, as long as the author is credited and the new creations are licensed under the identical terms

Accepted 20 June 2021

Revised 28 January 2021

Received 27 June 2020

Indian J Pharm Sci 2021;83(3):541-546 
commonly used for the treatment of renal insufficiencyinduced anemia in clinical practice. A pharmacological study proved that rhEPO can resist oxidation and apoptosis and promote nerve cell regeneration ${ }^{[6]}$. A recent study proved that rhEPO was able to protect brain tissues in TBI and had great potential in treating neurological diseases including stroke, cerebral hemorrhage and $\mathrm{TBI}^{[7]}$. In this study, therefore, rat models of TBI were established to investigate the mechanism of rhEPO in protecting neuronal cells in TBI from the perspective of effects of rhEPO on mitochondria and inflammatory responses, providing a basis for clinical experimental research in future.

\section{MATERIALS AND METHODS}

\section{Laboratory animals:}

A total of 60 specific-pathogen-free (SPF) grade male Sprague-Dawley (SD) rats aged 36-40 mo and weighing 280-320 g were provided by the Laboratory Animal Research Center of our hospital, adaptively fed in the animal room of our hospital at 18-25 and (56 \pm 5$)$ $\%$ humidity for $1 \mathrm{w}$ as per the administrative measures on laboratory animals of our hospital and then used in the experiments. During the experimental operations on animals in this study, the humane care for animals was performed in accordance with the 3Rs (Replacement, Reduction and Refinement) principle.

\section{Main reagents and apparatus:}

RhEPO was provided by Beijing Four Rings Biopharmaceutical Co., Ltd. (China), rhodamine 123 (Rh 123) stain was bought from Sigma and dynaminrelated protein 1 (Drp-1), fission 1 (Fis-1), mitofusin 2 (Mfn2) and optic atrophy 1 (Opa1) antibodies were purchased from Abcam (USA). Bicinchoninic acid (BCA) protein concentration determination kits and 3,3'-diaminobenzidine (DAB) chemistry luminescence kits were purchased from Nanjing Jiancheng Bioengineering Institute (China), interleukin-1 $\beta$ (IL$1 \beta$ ), interleukin-6 (IL-6) and tumor necrosis factor- $\alpha$ (TNF- $\alpha$ ) antibodies were provided by Cell Signaling Technology (CST) (USA) and sodium pentobarbital, an anesthetic for laboratory animals (subpackaged imports from Germany, $250 \mathrm{~g} / \mathrm{vial}$ ), paraformaldehyde and xylene were bought from Beijing Chemical Reagent Co., Ltd. (China). The frozen section embedding medium was purchased from Sakura (USA).

\section{Modeling and grouping:}

The rats were numbered and randomly assigned into sham operation group (sham group, $\mathrm{n}=15$ ), model group $(\mathrm{n}=15)$ and rhEPO intervention group (treatment group, $n=15$ ). In model group and treatment group, the rat model of TBI was established by hitting the head of rats through the modified Feeney's free-fall impact method. Specifically, the rats were anesthetized through intraperitoneal injection of pentobarbital sodium $(5 \%$, $0.15 \mathrm{ml} / 100 \mathrm{~g}$ ) and fixed in a prone position. Then, a longitudinal incision was made along the midline of the skull to expose the right parietal bone and a $5 \mathrm{~mm}$ diameter hole, $2 \mathrm{~mm}$ lateral to the sagittal suture, was drilled using a cranial drill. Next, a small hammer with a wounding force of $20 \mathrm{~g}$ was dropped from a height of $50 \mathrm{~cm}$ to create a brain contusion and laceration on the right side of the rat brain and the cerebral Dura mater of rats was kept intact. After that, hemostasis was done, followed by suture using a sterile surgical needle. The processing methods on laboratory animals in this study conformed to animal ethics standards. The above processing, except for hitting, was conducted on the rats in sham group. After operation, the rats in each group had varying degrees of limb twitching, poor balance and coordination of limbs, irregular breathing, mydriasis or miosis, weakened reaction of pupil to light and disappearance of eyelash reflexes, stinging reaction and corneal reflexes. Besides, oral or nasal hemorrhage or even temporary coma was observed in some rats. However, the results of behavioral observation showed that abnormal behaviors were recovered within $30 \mathrm{~min}$, suggesting successful modeling. After modeling, the rats in treatment group were given intraperitoneal injection of rhEPO at a dose of $5000 \mathrm{IU} / \mathrm{kg}$ daily and those in sham group and model group were intraperitoneally injected with equal dose of normal saline. Following continuous administration for $7 \mathrm{~d}$, the rats were decapitated to take their brains. Thereafter, the whole brain of rats in each group was fixed in $5 \%$ paraformaldehyde at room temperature for $48 \mathrm{~h}$. Afterwards, a $2 \mathrm{~mm}$ thick specimen was cut with the rat contusion as the center and a radius of $2 \mathrm{~cm}$, conventionally dehydrated, sealed in liquid nitrogen and stored in a cryogenic refrigerator for testing. During the experiment, 2 rats died in sham group, 3 died in model group and 1 died in treatment group. Finally, 15 rats were included in each group for statistical analysis.

\section{Detection of mitochondrial membrane potential (MMP) in brain tissues:}

The brain tissue specimen of various rats was taken out from the cryogenic refrigerator. Then, $100 \mathrm{mg}$ of specimens were weighed on an analytical balance, 
conventionally fixed and lysed and prepared into a single cell suspension. Next, the suspension was stained and washed according to the requirements of the Rh 123 kit, followed by observation using a US2018 fluorescence microscope. The green bright spots around the cell nucleus were the mitochondria taking in $\mathrm{Rh}$ 123. Thereafter, the green fluorescence intensity in the image was statistically analyzed using Image J 1.41.

\section{Observation of mitochondrial changes in brain tissue cells by transmission electron microscopy:}

After taking out the brain tissue specimen of various rats from the cryogenic refrigerator, $100 \mathrm{mg}$ was weighed on the analytical balance and prepared into ultra-thin tissue sections as follows: The specimen was fixed with $30 \%$ polyformaldehyde and $0.05 \%$ osmic acid for $1 \mathrm{~h}$. It was dehydrated under aseptic condition for $5 \mathrm{~min}$. It was placed in pre-cooled ethanol at $4^{\circ}$ for 3 min. It was soaked in epoxy resin 812 for 5 min and then embedded in paraffin. Following double staining with uranyl acetate and lead citrate, the specimen was sectioned into $50 \mathrm{~nm}$ slices. Thereafter, the slices are completely dried, loaded on a copper net and observed using the transmission electron microscope. Next, Simple-PCI 8.0 was employed for analysis and the specific surface area, volume density, number density and specific membrane area of mitochondria in rat brain tissue cells were detected.

\section{Determination of IL-1 $\beta$, IL-6 and TNF- $\alpha$ expression in tissue specimens by immunohistochemistry:}

A total of $100 \mathrm{mg}$ of brain tissue specimens taken out from the cryogenic refrigerator were weighed on the analytical balance. Then, according to the requirements of the immunohistochemistry kit, the specimen was subjected to fixation, embedding, sectioning, antigen retrieval, sealing and washing, incubated with primary antibodies and secondary antibodies, subjected to color development, counterstained, dehydrated and mounted, followed by observation under the microscope. The proportions of IL-1 $\beta$, IL- 6 and TNF- $\alpha$ positive cells were systematically analyzed through MetaMorph image software.

\section{Measurement of expression levels of Drp-1, Fis-1, Mfn2 and Opa1 by Western blotting:}

The brain tissue specimen of various rats was taken out from the cryogenic refrigerator. Then, $100 \mathrm{mg}$ of specimens were weighed on the analytical balance. Next, total proteins were routinely extracted, of which the concentration was determined. Thereafter,
$50 \mu \mathrm{g}$ of proteins were taken, loaded, subjected to gel electrophoresis and transferred to a membrane. Afterwards, the membrane was sealed and incubated with diluted primary antibodies (1:1000) respectively at $4^{\circ}$ overnight. The next day, the membrane was added with an appropriate amount of secondary antibodies (1:5000) and washed, followed by color development, photographing and grayscale scanning. Finally, the gray value of bands was analyzed using the ImageJ software and the relative expression was calculated with Glyceraldehyde 3-phosphate dehydrogenase (GAPDH) as the internal reference protein.

\section{Statistical analysis:}

The experimental data were statistically analyzed using SPSS 19.0 software. Normally distributed measurement data were expressed as mean \pm standard deviation $(\overline{\mathrm{x}} \pm \mathrm{s})$ and compared among groups through one-way analysis of variance and between groups by the independent t-test, $p<0.05$ suggested that difference was statistically significant.

\section{RESULTS AND DISCUSSION}

$\mathrm{Rh} 123$ fluorescent staining was carried out to detect the changes in MMP, which further reflected the integrity of cell mitochondrial structure. Compared with sham group, the Rh 123 fluorescence intensity of cells in brain tissues was significantly reduced in model group $(\mathrm{p}<0.05)$ and the mitochondrial membrane was severely damaged. The Rh 123 fluorescence intensity of cells in brain tissues was significantly stronger in treatment group than that in model group $(\mathrm{p}<0.05)$, implying that the mitochondrial injury in brain tissues of rats in treatment group is recovered to some extent (fig. 1).

A mitochondrion, a dynamic and relatively balanced organelle in the cell matrix of brain tissues, changes its shape mainly through the lysis and fusion of the

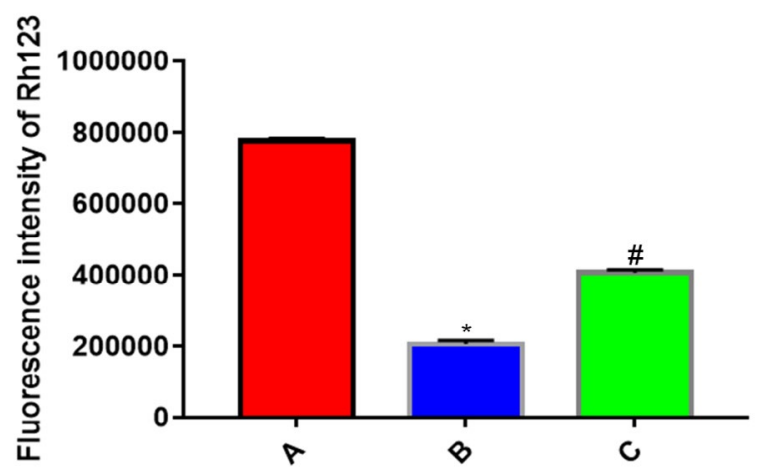

Fig. 1: Rh 123 staining results. A: sham group, B: model group and $C$ : treatment group, ${ }^{*} p<0.05$ vs. sham group, $\# p<0.05$ vs. model group 
inner and outer mitochondrial membranes, enabling its interconversion between the long interconnected network state and the disconnected broken state. Such a dynamic equilibrium process is called the dynamics of cell mitochondria in cell biology ${ }^{[8,9]}$. Drp-1 and Fis-1 are the major proteins modulating mitochondrial membrane lysis. The former is located on the mitochondrial membrane through the interaction with the adaptor protein Fis-1 to promote mitochondrial lysis. Mfn2 and Opa1 separately regulate the fusion of the outer and inner mitochondrial membranes.

To further clarify the protective effect of rhEPO on the mitochondria of TBI rats, the Western blotting was employed to detect the expression levels of Drp-1, Fis1, Mfn2 and Opa1 in brain tissues of each group of rats. The results showed that in comparison with sham group, model group had significantly increased expressions of Drp-1 and Fis-1 $(\mathrm{p}<0.05)$ and significantly lowered expressions of Mfn2 and Opa1 in rat brain tissues $(p<0.05)$. Compared with model group, treatment group displayed significantly reduced expressions of Drp-1 and Fis-1 $(\mathrm{p}<0.05)$ and significantly elevated expressions of Mfn2 and Opa1 in rat brain tissues $(\mathrm{p}<0.05)$ (fig. 2).

Mitochondrial changes in brain tissue cells are observed by transmission electron microscope. According to the stereological analysis of mitochondria in rat brain tissues in each group (fig. 3), the volume density, specific surface area, specific membrane area and number density of mitochondria in brain tissues were significantly smaller in model group than those in sham group $(\mathrm{p}<0.05)$, suggesting that the mitochondria in model group were seriously damaged. The above indices were significantly larger in treatment group
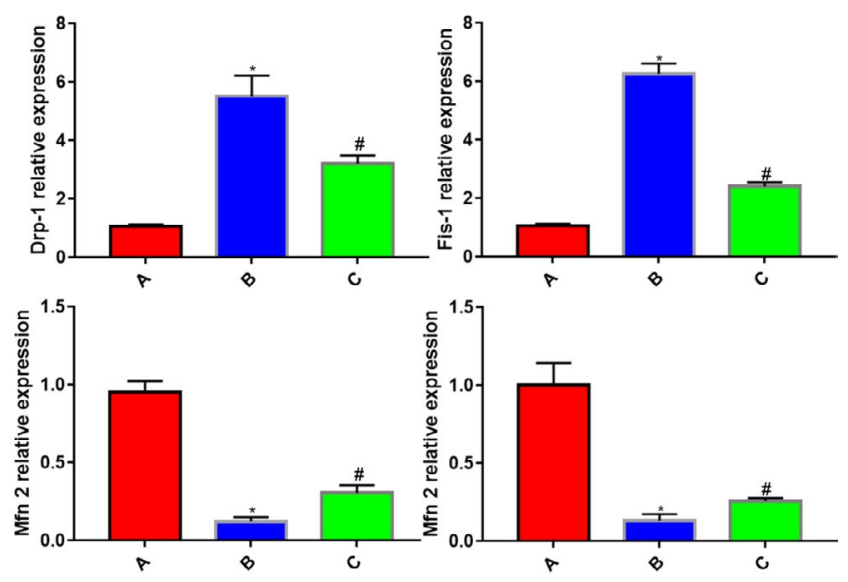

Fig. 2: Western blotting results. A: sham group, B: model group and $C$ : treatment group, ${ }^{*} p<0.05$ vs. sham group, \#p<0.05 vs. model group 


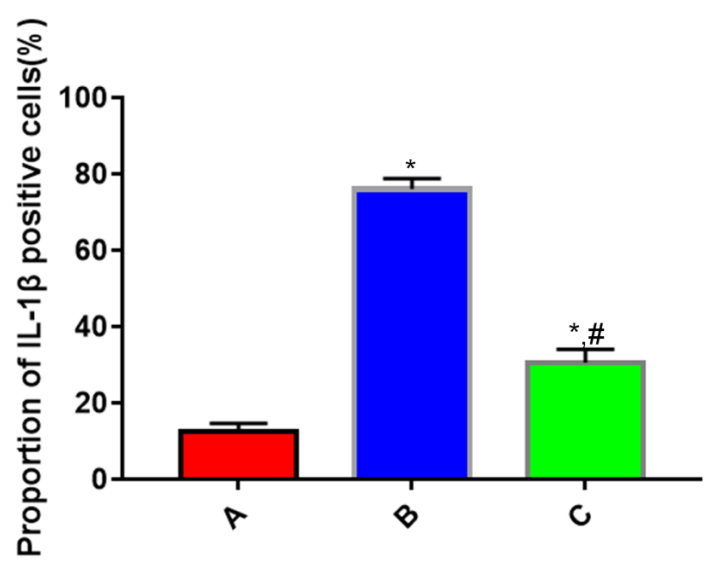

Fig. 4: IL-1ß expression detected by immunohistochemistry. A: sham group, B: model group and $\mathrm{C}$ : treatment group, " $\mathbf{p}<0.05$ vs. sham group, ${ }^{\#} \mathbf{p}<0.05$ vs. model group

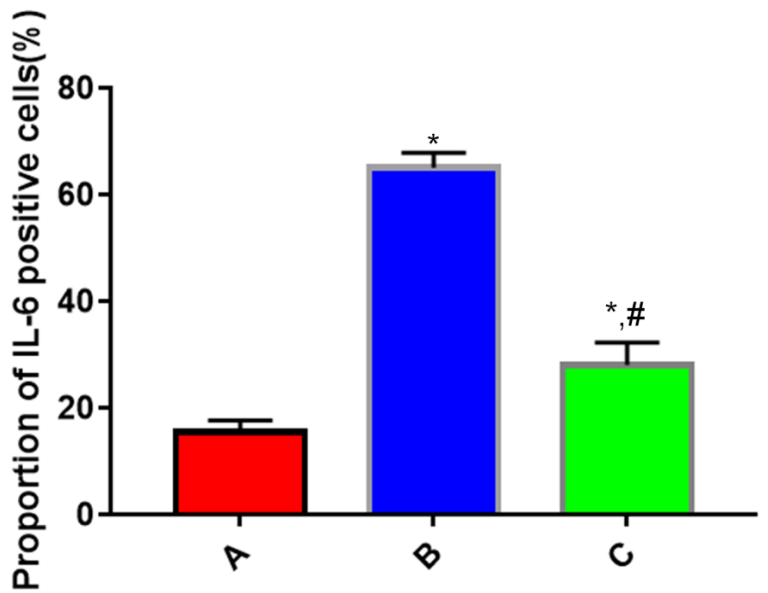

Fig. 5: IL-6 expression detected by immunohistochemistry. A: sham group, B: model group and $C$ : treatment group, " $p<0.05$ vs. sham group, ${ }^{\#} \mathbf{p}<0.05$ vs. model group

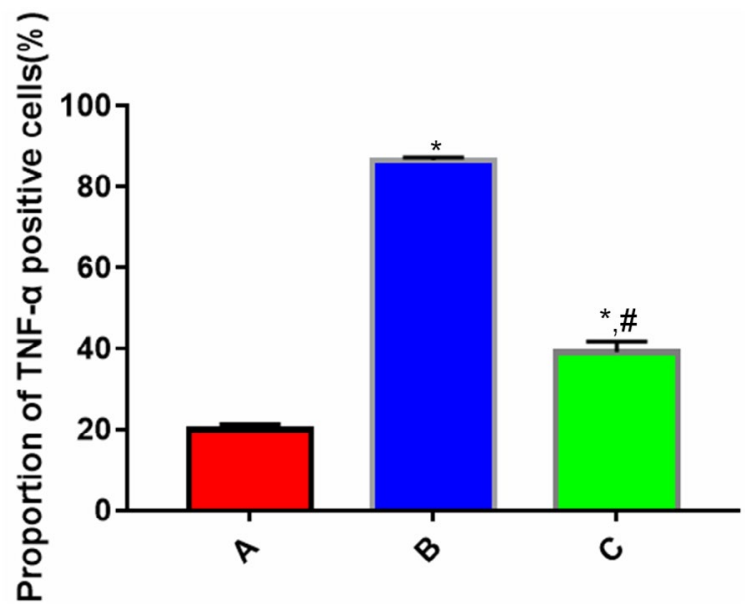

Fig. 6: TNF- $\alpha$ expression detected by immunohistochemistry. A: sham group, B: model group and $C$ : treatment group, " $p<0.05$ vs. sham group, ${ }^{\#} \mathbf{p}<0.05$ vs. model group

the model of $\mathrm{TBI}^{[14]}$. Mitochondria are the "power house" of cells and also the main places for energy metabolism of cells. Therefore, mitochondrial injury is the best indicator reflecting cellular lesions or injuries $^{[15]}$. In this study, the changes in MMP and the expression of mitochondrial dynamics-related proteins in brain tissues of rats in each group were separately detected through Rh 123 staining and Western blotting. Compared with sham group, model group exhibited significantly weakened Rh 123 fluorescence intensity of cells $(p<0.05)$, significantly increased expressions of Drp-1 and Fis-1 $(p<0.05)$ and significantly reduced expressions of Mfn 2 and Opa1 in brain tissues $(p<0.05)$, demonstrating severe mitochondrial injury in rat brain tissues in model group. In comparison with model group, treatment group had significantly enhanced $\mathrm{Rh} 123$ fluorescence intensity of cells $(\mathrm{p}<0.05)$, significantly reduced expressions of Drp-1 and Fis$1(p<0.05)$ and significantly elevated expressions of Mfn2 and Opa1 in brain tissues $(p<0.05)$, implying certain recovery of mitochondrial injury in rat brain tissues in treatment group. Besides, the changes in the ultrastructure of mitochondria were observed using the electron microscope and stereological analysis was carried out, so as to further study the mitochondrial injury. The results revealed that the volume density, specific surface area, specific membrane area and number density of mitochondria in brain tissues were significantly smaller in model group than those in sham group ( $\mathrm{p}<0.05)$, suggesting severe mitochondrial injury in model group. The above indices were significantly larger in treatment group than those in model group $(p<0.05)$, indicating certain recovery of mitochondrial injury in treatment group.

During the progression of TBI, the inflammatory response plays a vital role and IL- $1 \beta$, IL- 6 and TNF- $\alpha$ are the most common inflammatory factors. RhEPO can significantly inhibit the inflammatory response of the heart, lungs and other organs after injury ${ }^{[16]}$. However, the relationship between rhEPO and inflammatory response after brain injury is not reported. In this study, the expression of inflammatory factors was measured via immunohistochemistry and Western blotting. The results uncovered that the expressions of IL-1 $\beta$, IL- 6 and TNF- $\alpha$ in rat brain tissues were significantly higher in model group than those in sham group $(p<0.05)$, whereas they were significantly lower in treatment group than those in model group $(\mathrm{p}<0.05)$, suggesting that rhEPO is capable of significantly repressing the inflammatory response after TBI.

In conclusion, rhEPO can protect the brain after TBI by relieving the inflammatory response and mitochondrial injury after TBI. However, further exploration should be carried out on how to apply the protective effect of 
rhEPO to clinical targeted treatment of cerebrovascular diseases after TBI.

\section{Author's contributions:}

Kai Xue and Zheng Jin contributed equally to this study.

\section{Acknowledgements:}

This study was financially supported by Tianjin health and Health Committee Science and Technology Project (No. QN20009).

\section{Conflicts of interest:}

The authors declared no conflict of interest.

\section{REFERENCES}

1. Metcalf K, Sabaz M, Daher M, Simpson G. Measuring reliable change in traumatic brain injury (TBI): The pitfalls of using readily available formulae. Appl Neuropsychol Adult 2020;27(5):421-30.

2. Kinder HA, Baker EW, West FD. The pig as a preclinical traumatic brain injury model: current models, functional outcome measures and translational detection strategies. Neural Regen Res 2019 Mar;14(3):413-24.

3. Chen X, Wang H, Zhou M, Li X, Fang Z, Gao H, et al. Valproic acid attenuates traumatic brain injury-induced inflammation in vivo: involvement of autophagy and the Nrf2/ARE signaling pathway. Front Mol Neurosci 2018;11:117.

4. Hinson HE, Rowell S, Schreiber M. Clinical evidence of inflammation driving secondary brain injury: a systematic review. J Trauma Acute Care Surg 2015;78(1):184-91.

5. Thelin EP, Hall CE, Gupta K, Carpenter KL, Chandran S, Hutchinson $\mathrm{PJ}$, et al. Elucidating pro-inflammatory cytokine responses after traumatic brain injury in a human stem cell model. J Neurotrauma 2018;35(2):341-52.

6. Vestergaard MB, Henriksen OM, Lindberg U, AachmannAndersen NJ, Lisbjerg K, Christensen SJ, et al. No evidence for direct effects of recombinant human erythropoietin on cerebral blood flow and metabolism in healthy humans. J Appl Physiol 2018;124(4):1107-16.

7. Bai XF, Gao YK. Recombinant human erythropoietin for treating severe traumatic brain injury. Medicine 2018;97(1):e9532.

8. Hung CH, Cheng SS, Cheung YT, Wuwongse S, Zhang NQ, Ho YS, et al. A reciprocal relationship between reactive oxygen species and mitochondrial dynamics in neurodegeneration. Redox Biol 2018;14:7-19.

9. Wang H, Yi J, Li X, Xiao Y, Dhakal K, Zhou J. ALS-associated mutation SOD1G93A leads to abnormal mitochondrial dynamics in osteocytes. Bone 2018;106:126-38.

10. Evans LP, Newell EA, Mahajan M, Tsang SH, Ferguson PJ, Mahoney $\mathrm{J}$, et al. Acute vitreoretinal trauma and inflammation after traumatic brain injury in mice. Ann Clin Transl Neurol 2018;5(3):240-51.

11. Huijben JA, Volovici V, Cnossen MC, Haitsma IK, Stocchetti $\mathrm{N}$, Maas AI, et al. Variation in general supportive and preventive intensive care management of traumatic brain injury: a survey in 66 neurotrauma centers participating in the Collaborative European NeuroTrauma Effectiveness Research in Traumatic Brain Injury (CENTER-TBI) study. Crit Care 2018;22(1):1-9.

12. Liu WC, Wen L, Xie T, Wang H, Gong JB, Yang XF. Therapeutic effect of erythropoietin in patients with traumatic brain injury: a meta-analysis of randomized controlled trials. J Neurosurg 2016;127(1):8-15.

13. Irimia A, Van Horn JD. Functional neuroimaging of traumatic brain injury: advances and clinical utility. Neuropsychiatr Dis Treat 2015;11:2355-65.

14. Yang Y, Wei H, Zhou X, Zhang F, Wang C. Hyperbaric oxygen promotes neural stem cell proliferation by activating vascular endothelial growth factor/extracellular signal-regulated kinase signaling after traumatic brain injury. Neuroreport 2017;28(18):1232-8.

15. VanderVeen BN, Fix DK, Carson JA. Disrupted skeletal muscle mitochondrial dynamics, mitophagy and biogenesis during cancer cachexia: a role for inflammation. Oxid Med Cell Longev 2017;2017: 3292087.

16. Blixt J, Gunnarson E, Wanecek M. Erythropoietin attenuates the brain edema response after experimental traumatic brain injury. J Neurotrauma 2018;35(4):671-80. 